\title{
Nightmare-Enacting Behavior Responding to Zonisamide in Early Parkinson's Disease
}

\author{
Hiroshi Kataoka Satoshi Ueno \\ Department of Neurology, Nara Medical University, Kashihara, Japan
}

\section{Key Words}

Zonisamide $\cdot$ Parkinson $\cdot$ Dream $\cdot$ Nightmare $\cdot$ REM sleep behavior disorder

\begin{abstract}
Recently, zonisamide (ZNS) has been approved as a new adjunctive therapy for motor complications of Parkinson's disease (PD). More recently, ZNS was reported to be effective for the management of impulse control behavior in PD, suggesting potential effects on nonmotor PD symptoms. Dream enactment associated with aggressive, violent behavior can carry a serious risk of injury to patients, as well as to spouses or caretakers. This report describes a patient with PD who had vivid nightmares and dream-enacting behavior that resolved after treatment with ZNS. The present case raises the question whether ZNS might potentially be effective for the management of vivid nightmares or dream-enacting behavior.
\end{abstract}

\section{Introduction}

Zonisamide (ZNS) is an antiepileptic drug that has recently been approved in many countries. It is also effective for the management of migraine [1], neuropathic pain [2], essential tremor [3], anxiety [4], and other conditions. Recently, ZNS has been approved as a new adjunctive therapy for motor complications of Parkinson's disease (PD) in Japan [5]. More recently, ZNS was reported to be effective for the management of impulse control behavior in PD [6], suggesting potential effects on non-motor PD symptoms. Dream enactment associated with aggressive, violent behavior can carry a serious risk of injury to patients, as well as to spouses or caretakers. We describe a patient with PD who had vivid nightmares and dream-enacting behavior that resolved after treatment with ZNS. 


\section{Case Report}

In 2009, a 71-year-old man with a history of pulmonary surgery noticed tremor of the left hand and akinesia. In March 2010, he showed features of moderate parkinsonism, including masked face, stooped posture, bradykinesia, left-side-dominant rigidity, and resting tremor. Cranial nerve, sensory, and cerebellar functions were intact. The results of cranial magnetic resonance imaging were normal. Bradykinesia, rigidity, and resting tremor responded to treatment with pramipexole $(0.5 \mathrm{mg} / \mathrm{day})$. Since July 2010, his wife was awakened nearly every night because of the patient's aggressive or violent behavior during the middle of the night. For example, he suddenly flew up or walked, collided with furniture or walls, cried out, or exercised his limbs noisily. He was often injured. On the nights he presented with this behavior, he remembered having vivid nightmares, such as being chased by a cat, bear, or his wife or of fighting with a thief or grandchild. These nightmares were documented in personal interviews. Interviews with the patient's wife indicated that this aggressive, violent behavior during the night occurred sometimes in 2009. In April 2011, stooped posture and lumbar pain developed, which were attributed to pramipexole. We switched from pramipexole $(1.0 \mathrm{mg} / \mathrm{day}$, once before sleep) to ZNS (25 mg/day, once before sleep). Before switching to ZNS, motor and non-motor symptoms were as follows: the scores on parts I, II, III, and IV of the unified Parkinson's disease rating scale (UPDRS) were 3, 5, 17, and 0, respectively. The scores on the Mini-Mental State Examination (29/30) and Frontal Assessment Battery (18/18) were normal. The heart-mediastinum 123Imetaiodobenzylguanidine uptake ratio was significantly decreased. Because he noticed reduced olfactory sensitivity, we assessed olfactory recognition ability by using the Odor Stick Identification Test for Japanese (OSIT-J), as described previously [7]. Briefly, the OSIT-J tests for 13 kinds of odors familiar to Japanese people (curry, cooking gas, perfume, Japanese cypress, India ink, menthol, nattou [fermented soybeans]/sweaty socks, rose, putrid smell, wood, roasted garlic, condensed milk, Japanese orange). We asked the patient to choose an answer among four possible odor names, one of which was correct, plus 'detectable but not recognizable' and 'odorless'. He could identify four odors (perfume, curry, cooking gas, and condensed milk). The identification rate was $33 \%$, consistent with that of patients with olfactory disturbances (36 $\pm 34 \%$ ). The score on the Pittsburgh sleep quality index (ranging from 14 for normal to 56 for unsleeping) was 16, and the 2-point increase above normal was ascribed to difficulty in sleeping caused by nightmares. He fell asleep in 5 minutes and slept for $7 \mathrm{~h}$ without sleep fragmentation. He did not experience hallucinations or pleasant dreams during follow-up. In May, he could never remember his dreams, and the nightmares disappeared. His wife was no longer awakened since the patient's aggressive and violent behavior had resolved. Motor features, including the UPDRS score, were unchanged. This state has persisted for 7 months. In November 2011, we obtained informed consent from the patient and tested whether pramipexole elicited nightmares or violent behavior. First, he was given pramipexole $(0.5 \mathrm{mg} /$ day $)$ in the context of stable doses of ZNS ( $25 \mathrm{mg}$ /day) for 2 weeks. Subsequently, ZNS was withdrawn, and pramipexole was continued for 2 weeks. No nightmares or violent behavior appeared.

\section{Discussion}

Our patient showed aggressive and violent behavior associated with vivid nightmares, which probably preceded such behavior, during the middle of the night. He did not have a history of hallucinations. This disorder strongly suggested a rapid-eyemovement sleep behavior disorder (RBD). In our patient, who had RBD while receiving pramipexole, RBD was completely resolved by switching from pramipexole to ZNS. Dopamine agonists can lead to vivid dreams, nightmares, and parasomnia-like motor activity [8], but RBD in our patient was not induced by rechallenge with pramipexole. In patients with PD, pramipexole was reported to be effective for RBD [9], but to worsen REM sleep electromyographic abnormalities on video-polysomnography [10]. One study showed that pramipexole did not improve RBD in PD, and the authors mentioned that RBD in PD may be either 2 different stages of the same condition or 2 completely different conditions [11]. This can explain our observations during treatment with pramipexole, but we believe that pramipexole did not alter RBD in our patient since the wife's interviews indicated that RBD was evident before starting 
pramipexole. This notion is supported by the results of a previous study [11] showing that the frequency and severity of RBD were unaffected by pramipexole therapy. RBD itself can disappear in the natural history of PD [12]. However, our finding raises an open question whether ZNS is useful for treating RBD in patients with early PD.

Abnormal dreams in RBD contribute to the control of enacted behavior [13]. Dream generators are suppressed by inhibition of brainstem locomotor pattern generators, which can modify dream content in RBD [13]. Dopaminergic dysfunction may play a role in the pathophysiology of RBD [14] and dopamine modulates the expression of locomotion and other rhythmic motor patterns in neural circuits known as central pattern generators [15]. A ZNS-induced effect on the dopaminergic system might have modulated or inhibited brainstem locomotor pattern generators, consequently suppressing vivid nightmares, leading to the resolution of dream-enacting behavior. Our experience suggests that ZNS potentially might be effective for the management of vivid nightmares or dream-enacting behavior in patients with early PD.

\section{Disclosure Statement}

The authors report no conflicts of interest. There was no financial disclosure related with this work.

\section{References}

1 Bermejo PE, Dorado R: Zonisamide for migraine prophylaxis in patients refractory to topiramate. Clin Neuropharmacol 2009;32:103-106.

$\checkmark 2$ Atli A, Theodore BR, Turk DC, Loeser JD: Zonisamide in the treatment of painful diabetic neuropathy: a randomized, double-blind, placebo-controlled pilot study. Pain Med 2010;11:1010-1016.

-3 Bermejo PE, Ruiz-Huete C, Dorado R, Anciones B: Zonisamide in refractory essential tremor. Rev Neurol 2008;46:139-142.

-4 Kinrys G, Vasconcelos e Sa D, Nery F: Adjunctive zonisamide for treatment refractory anxiety. Int J Clin Pract 2007;61:1050-1053.

-5 Murata M, Hasegawa K, Kanazawa I; Japan Zonisamide on PD Study Group: Zonisamide improves motor function in Parkinson disease: a randomized, double-blind study. Neurology 2007;68:45-50.

-6 Bermejo PE, Ruiz-Huete C, Anciones B: Zonisamide in managing impulse control disorders in Parkinson's disease. J Neurol 2010;257:1682-1685.

-7 Kobayashi M: The Odor Stick Identification Test for the Japanese (OSIT-J): clinical suitability for patients suffering from olfactory disturbance. Chem Senses 2005;30(suppl 1):i216-i217.

-8 Pagel JF, Helfter P: Drug induced nightmares - an etiology based review. Hum Psychopharmacol 2003;18:59-67.

-9 Aurora RN, Zak RS, Maganti RK, Auerbach SH, Casey KR, Chowdhuri S, Karippot A, Ramar K, Kristo DA, Morgenthaler TI; Standards of Practice Committee; American Academy of Sleep Medicine: Best practice guide for the treatment of REM sleep behavior disorder (RBD). J Clin Sleep Med 2010;6:85-95.

10 Fantini ML, Gagnon JF, Filipini D, Montplaisir J: The effects of pramipexole in REM sleep behavior disorder. Neurology 2003;61:1418-1420.

11 Kumru H, Iranzo A, Carrasco E, Valldeoriola F, Marti MJ, Santamaria J, Tolosa E: Lack of effects of pramipexole on REM sleep behavior disorder in Parkinson disease. Sleep 2008;31:1418-1421.

12 Gjerstad MD, Boeve B, Wentzel-Larsen T, Aarsland D, Larsen JP: Occurrence and clinical correlates of REM sleep behaviour disorder in patients with Parkinson's disease over time. J Neurol Neurosurg Psychiatry 2008;79:387-391.

13 Schenck CH, Mahowald MW: A polysomnographic, neurologic, psychiatric, and clinical outcome report on 70 consecutive cases with REM sleep behavior disorder (RBD): sustained clonazepam efficacy in 89.5\% of 57 treated patients. Clev Clin J Med 1990;57:S9-S23.

-14 Eisensehr I, Linke R, Noachtar S, Schwarz J, Gildehaus FJ, Tatsch K: Reduced striatal dopamine transporters in idiopathic rapid eye movement sleep behaviour disorder. Comparison with Parkinson's disease and controls. Brain 2000;123:1155-1160.

15 Harris-Warrick RM, Marder E: Modulation of neural networks for behavior. Annu Rev Neurosci 1991;14:39-57. 